\title{
PERFORMANCE CHARACTERISTICS OF THE HIGH POWER DENSITY, FLUX-CONSERVING TOKAMAK (FCT) REACTOR†
}

\author{
S. K. BOROWSKI and TERRY KAMMASH \\ The University of Michigan, Ann Arbor, MI 49109, U.S.A.
}

(Received 7 November 1978)

\begin{abstract}
Most of the fusion Experimental Power Reactor (EPR) and commercial power reactor designs have been limited to low- $\beta$ (ratio of plasma pressure to magnetic field pressure), low power density Tokamak systems. In an effort to redesign a viable, economically attractive power reactor that could meet the electric utilities needs, the flux-conserving Tokamak concept bas emerged as a leading contender.

In this paper, we examine the operating characteristics of an FCT reactor and show that such a system would be comparable in size to an EPR but produce more than five times the fusion power. It will be seen that the FCT concept could provide the utility industry with compact reactor systems of moderate electrical output (between 500 and $1000 \mathrm{MWc}$ ) without requiring large extrapolations in plasma size and technology beyond that of the Tokamak Fusion Test Reactor (TFTR) currently under construction.
\end{abstract}

\section{INTRODUCTION}

One of the major problems of low beta (plasma to magnetic pressure) tokamak reactors is the associated low power density that might translate into large and perhaps prohibitively expensive power producing systems. Although the use of non-circular cross sections have been shown to result in some enhancement in the value of beta a more dramatic improvement can be expected to occur if the reactor is operated in a flux conserving mode. ${ }^{1}$ Recent numerical investigations ${ }^{2}$ of plasma equilibria in such systems have shown that values of total beta of $10-20 \%$ are feasible although more realistic beta limits in the range 5-10\% may be dictated by the onset of ballooning instability.

In supplementing a larger study, ${ }^{3}$ addressing the impact of confinement physics on fusion reactor design and economics, this paper reports on the results of a global analysis for a possible prototype power producing tokamak reactor operating in a flux conserving mode. Transport loss is computed from combined scaling laws, based on pseudo-classical, neoclassical, and dissipative trapped particle mode scaling, and initial plasma heating by neutral beam injection is utilized. A combination of low density startup and controlled fuel density buildup is used to partially alleviate beam penetration problems while allowing for the enhanced alpha heating needed for ignition.

Analysis indicates that such a system would be comparable in size to an EPR ${ }^{4}$ but produce more than five times the fusion power considered feasible for that design concept. The FCT concept could provide the electric utility industry with compact reactor systems of moderate electrical output (between 500 and $1000 \mathrm{MWe}$ ) without necessitating large extrapolations both in plasma size and technology beyond that of the Tokamak Fusion Test Reactor (TFTR).

\section{BASIC EQUATIONS AND ANALYSIS}

In the plasma calculational model used in this study the $D-T$ ions are treated as a single species with an effective mass (a.m.u.) $A_{D T}=3 p+2(1-p)$ where $p$ denotes the fraction of the D-T ions which is tritium. Global balance equations ${ }^{5}$ are used where it is assumed that the alpha particles and the injected energetic deuterons thermalize instantaneously and distribute their energy among the electrons, the D-T ions, the thermalized alphas and any impurity ions which may be present. It is noted here that in slowing down the injected deuterons and fusion alphas give up a total energy to the bulk plasma equal to $U_{0}-3 / 2 T_{b}$, where $U_{0}$ is the initial energy of the test particle. The power produced by the injected deuterons is augmented by the alpha heating resulting from suprathermal fusion of the fast deuterons with the thermal tritons.

†This paper was presented at the 1978 Midwest Energy Conference, Chicago, Illinois, 19-21 November, 1978.

‡Work supported by EPRI and DOE. 
Particles are lost from the plasma by diffusion and fusion while power is lost through radiation and transport. The equations of interest are given in M.K.S. units with $T$ in $\mathrm{keV}$ as follows:

$$
\begin{gathered}
\frac{\mathrm{d} n_{i}}{\mathrm{~d} t}=I_{c}+I_{B}(1-2 f)-2 p(1-p) n_{1}^{2}\langle\sigma v\rangle-\frac{n_{i} n_{e}}{\left(n_{e} \tau\right)_{p}}, \\
\frac{3}{2} \frac{\mathrm{d}}{\mathrm{d} t}\left(n_{i} T_{i}\right)= \\
p(1-p) n_{i}^{2}\langle\sigma v) U_{\alpha} f_{\alpha i}+G_{B} I_{B} U_{B} \hat{f}_{B i} \\
+\frac{3}{2} T_{i}\left[I_{B}(1-2 f)-2 p(1-p) n_{i}^{2}\langle\sigma v\rangle\right]-K_{i e}-\frac{3}{2} T_{i} \frac{n_{i} n_{e}}{\left(n_{e} \tau\right)_{E i}} \\
\frac{\mathrm{d} n_{\alpha}}{\mathrm{d} t}=p(1-p) n_{i}^{2}\langle\sigma v\rangle+f I_{B}-\frac{n_{e} n_{\alpha}}{\left(n_{e} \tau\right) p} . \\
n_{e}=n_{i}+2 n_{\alpha}+\sum n_{I} Z_{I}, \\
\frac{3}{2} \frac{\mathrm{d}}{\mathrm{d} t}\left(n_{e} T_{e}\right)=p(1-p) n_{i}^{2}\langle\sigma v) U_{\alpha} f_{\alpha e}+G_{B} I_{B} U_{B} \hat{f}_{B e}+\eta J^{2}+K_{i e}-W_{R}-\frac{3}{2} T_{e} \frac{n_{e}^{2}}{\left(n_{e} \tau\right)_{E e}} .
\end{gathered}
$$

In the above equation $I_{c}$ is the cold D-T source, $I_{B}$ is the injected source of energetic deuterons, $\langle\sigma v\rangle$ is the Maxwellian averaged D-T cross section, $\tau_{p}$ is the particle confinement time, $\tau_{E i}$ and $\tau_{E e}$ are the ion and electron energy confinement time, respectively, and $f$ is the fraction of the beam which undergoes suprathermal fusion. The quantity $G_{B}$ defined by

$$
G_{B}=\frac{U_{B}+f U_{\alpha}}{U_{B}}
$$

represents the suprathermal beam enhancement factor with $U_{\alpha}=3.5 \mathrm{MeV}$, and $U_{B}$ denoting the energy of the injected dueterons; while $\hat{f}_{B j}$ is given by

$$
\hat{f}_{B j}=\frac{U_{B} f_{B j}+f U_{\alpha} f_{\alpha i}}{G_{B} U_{B}} ; j=i, e
$$

in which $f_{B j}$ and $f_{\alpha j}$ denote respectively the fraction of the beam and alpha energy given to the D-T ions and electrons. In the electron energy balance balance equation, the terms $\eta J^{2}, K_{i e}$, and $W_{R}$ represent ohmic heating, electron cooling on the ions and radiation loss, respectively.

In order to solve the above balance equations it is necessary to calculate the appropriate particle and energy confinement times. The confinement scenario used in this simulation computes "weighted" particle and energy confinement times ${ }^{6}$ based on pseudoclassical, neoclassical, and dissipative trapped particle mode scaling laws. The "weighting effect" takes into account the dominance of a particular diffusion mode over the other modes as the plasma evolves through various regimes of collisionality. During the burn cycle, the steady-state operation of the reactor is characterized by confinement times which are assumed to be ten times the trapped particle modes.

\section{PLASMA HEATING TO FLUX CONSERVING CONDITIONS}

Recent theoretical studies' have indicated that the initiation of high power neutral beam heating can lead to the natural production of high- $\beta$ plasma equilibria provided beam heating can increase the stored energy in the plasma rapidly i.e., if the beam heating time, $\tau_{h}$, is considerably less than the bulk plasma energy confinement time, $\tau_{\mathrm{E}}$. The result of such rapid heating is that a transient magnetic flux diffusion profile (its initial resistive decay resulting in ohmic heating) will be slowed and eventually "frozen" into the plasma since $\tau_{h}$ is much shorter than the flux diffusion time scale given by (in C.G.S. units)

$$
\tau_{m}=\frac{2 \pi L^{2}}{\eta c^{2}}
$$


where $L$ is a characteristic length of the spatial variation in the magnetic field and $\eta$ is the plasma resistivity. Since the toroidal and poloidal fluxes are frozen into and, therefore, preserved in the plasma, it should be possible to operate at a significantly higher equilibrium plasma pressure. The earlier equilibrium and plasma confinement limitations associated with an inwardly moving magnetic ${ }^{\prime}$ separatrix are avoided ${ }^{7}$ and the familiar limitations on poloidal beta $\left(\beta_{p} \leqslant A\right)$ are removed allowing $\beta$ to increase monotonically with $\beta_{p}$. Flux conservation also results in a freezing of the $q$-profile as the high beta equilibria evolve. As a result, it appears that these FCT equilibria will exhibit the same $q$ (safety factor) profile as the low beta equilibria which exists prior to the commencement of beam heating and should, therefore, possess reasonable stability properties against local MHD modes. ${ }^{7}$ In principle then, the flux conserving mode of operation can lead to transient higher $\beta$ equilibria which for moderately-sized systems (plasma radii ranging from 1 to 2 meters), can last for hundreds of seconds, i.e. on the order of the magnetic diffusion time.

Although the results which follow indicate that FCTs can support high $\beta$, high density operation, several areas require further study before the true potential of FCTs can be assessed. These areas are outlined below.

\section{(1) Duration of FCT equilibria}

Following the heating phase, it is expected that the FCT high $\beta$ equilibrium will resistively decay on the long magnetic diffusion time scale of the hot plasma. The possibility of anomalous flux diffusion could result in an appreciable deterioration of the anticipated magnetic diffusion time. Studies ${ }^{8}$ have indicated that it may be possible to maintain the high $\beta$ equilibrium beyond the skin time by reducing the plasma resistivity in regions where high density is required for high $\beta$. The ECR heating technique, with variable frequency capabilities, appears particularly attractive since localized heating in a narrow resonance zone is possible through the proper selection of frequency.

\section{(2) Beam penetration}

As discussed above, the ability to increase the stored energy in the plasma rapidly is essential to the natural production of high- $\beta$ FCT plasma equilibria. As the size of the plasma increases, the ability to successfully penetrate to the core of a high density plasma becomes less effective. The neutral beam deposition profiles shown in Fig. 1 give the plasma target thickness $a_{p} n_{e}(0)$ as a function of particle injection energy for various values of $Z_{\text {eff. }}$ These profiles provide us with a rough estimate of the maximum penetrable density for the FCT system under consideration for the case of tangential injection. For a plasma radius of $1.50 \mathrm{~m}$ with $200 \mathrm{keV}$ deuteron beams, Fig. 1 indicates marginal penetration for central core densities in excess of approx. $1.3 \times 10^{20} / \mathrm{m}^{3}$ for $Z_{\text {eff }} \simeq 1$. As will be shown in Section 5 , a low-density startup technique appears attractive for circumventing this problem.

\section{(3) Plasma control}

Plasma control during burn and especially during shutdown is essential due to the large thermal and magnetic energy content of the ignited plasma. Approximately 550 megajoules in kinetic energy content and approx. 90 megajoules $\left(=1 / 2 L_{p} I_{p}^{2}\right)$ in magnetic energy are contained in a FCT power reactor.

It is noted here, however, that problem areas (2) and (3), in addition to the mutual problem of acceptable limits on wall loading, are also common to the noncircular high- $\beta$ plasma concept which itself has inherent problems such as increased $q$-values necessary for stability and increased systems cost attributed to the complex field-shaping coils.

\section{FCT REACTOR SCALING}

The parameters for the FCT reactor analyzed in this study along with the injection heating power requirements are based on extrapolation from the ORNL/TNS Reference Design ${ }^{10}$ which has a plasma radius of $1.25 \mathrm{~m}$. Maintaining the same elongation safety factor, aspect ratio and plasma current, the plasma radius is increased to $1.50 \mathrm{~m}$, with a concomitant increase in the plasma volume by a factor of approx. 2. The toroidal magnetic field on axis needed for such a 


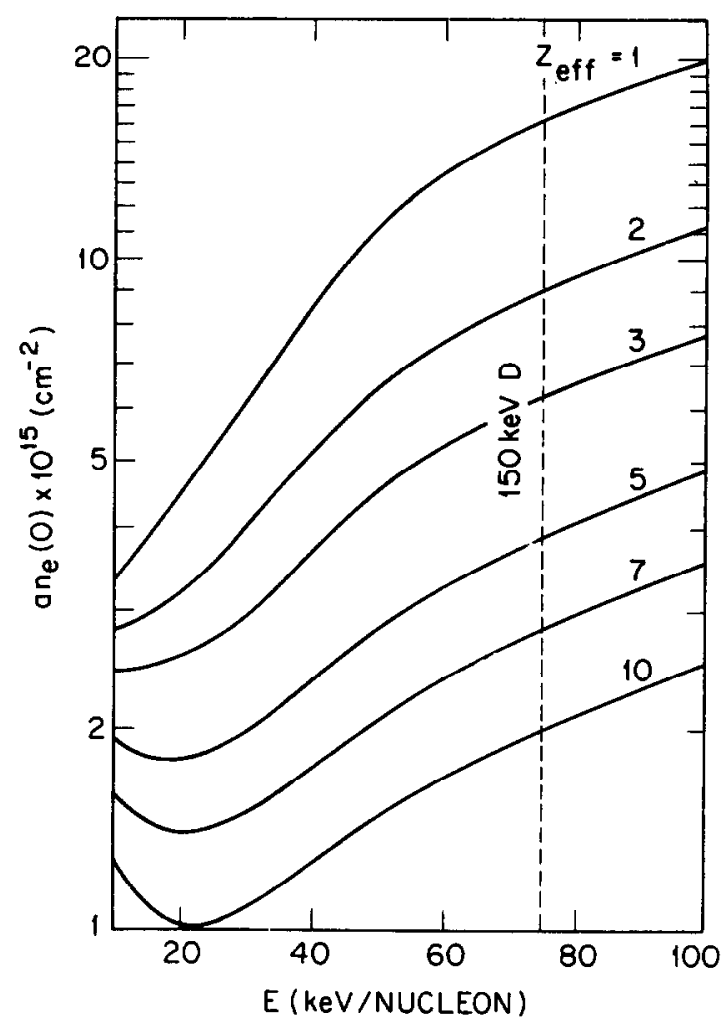

Fig. 1. Maximum plasma density for beam penetration times plasma radius as a function of beam energy per nucleon with $Z_{\text {eff }}$ as a parameter. Note that the energy required for deuterium is twice that for hydrogen injection (Ref. 9).

device can be estimated directly from the expression for the plasma "safety factor" appropriately modified for a non-circular plasma as follows:

$$
B_{T}=\frac{\mu_{0} I_{p} q(a) A}{2 \pi a}\left(\frac{2}{1+\sigma^{2}}\right)
$$

For $a=1.50 \mathrm{~m}$ one finds that the required magnetic field is $B_{T}\left(R_{0}\right)=3.6$ Tesla. Similarly the maximum field at the surface of the superconducting coil can be estimated using the expression for the toroidal magnetic field on axis which is given by

$$
B_{T}\left(R_{0}\right)=B_{T}^{\operatorname{MAX}}\left\{1-\left[\frac{1+\Delta / a}{A}\right]\right\}
$$

where $A$ is the aspect rate, $a$ is the plasma radius and $\Delta$ is the distance between the inner plasma edge and the inner surface of the toroidal field coil which includes space for plasma scrape off, shielding and blanket. For $B_{T}\left(R_{0}\right)=3.6$ Tesla and $\Delta / a \sim 1$, Equation (10) indicates that the maximum field at the surface of the superconducting coil is $B_{T}^{\mathrm{MAX}} \simeq 7.2 \mathrm{Tesla}$, well within the limits for $\mathrm{NbTi}$ superconducting coils. The reactor parameters for both the $1.25 \mathrm{~m}$ ORNL/TNS Reference design and the $1.50 \mathrm{~m}$ FCT reactor are summarized in Table 1.

\section{BURN DESCRIPTION}

Simulation of the FCT prototype power reactor burn begins after the $D-T$ fuel gas has been ionized and heated to a temperature of about $100 \mathrm{eV}$. The initial $D-T$ ion density is taken to be $7.5 \times 10^{19} / \mathrm{m}^{3}$ and a $Z_{\text {eff }}$ of about unity. During the first second of operation the plasma current is ramped up to its low beta value of 4.0 MA, and a relatively hot $(\sim 0.35 \mathrm{keV})$ ohmically heated plasma is produced $(\sim 0.50 \mathrm{keV}$ for $2 \mathrm{sec}$ of ohmic heating). At $1 \mathrm{sec}$, injection of $100 \mathrm{MW}$ of supplemental neutral beam power in the form of $200 \mathrm{keV}$ deuterons is initiated. Once the beam is turned on, plasma heating becomes rapid proceeding at a rate of $\sim 7.0 \mathrm{keV}$ per sec until the 
Table 1. FCT reactor parameters.

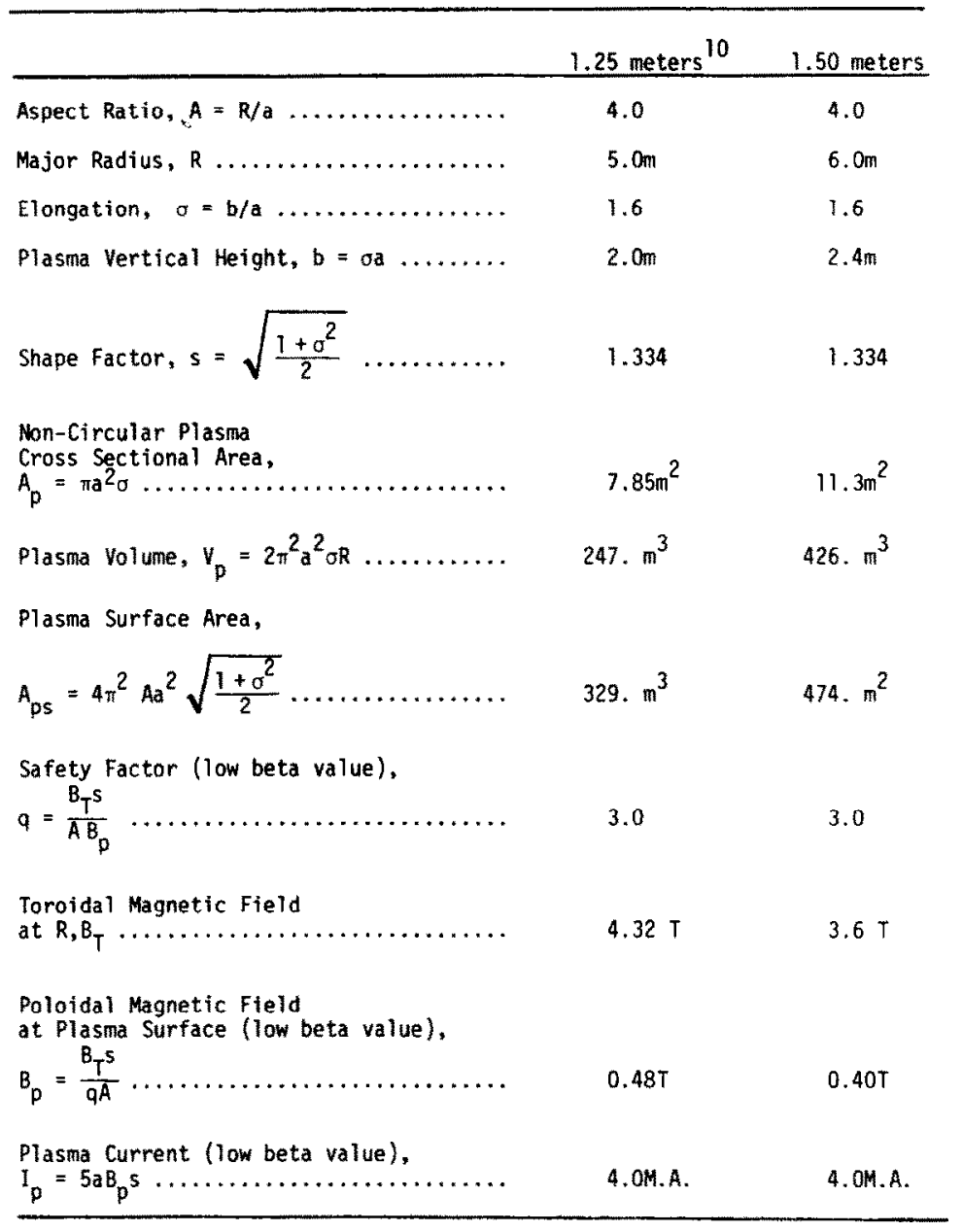

ion temperature reaches $10 \mathrm{keV}$. At this temperature the plasma density is increased. The surge of cold refuel produces some temporary thermal fluctuations which are quickly overcome by the combined beam and fusion alpha heating. At $4.2 \mathrm{sec}$ the steady-state operating density is reached with sufficient power margin $\left(p_{\alpha}-p_{\text {Loss }}^{\text {Tot }}=40 \mathrm{MWs}\right)$ for ignition to occur. Following termination of the supplemental beam heating is a gradual climb resulting from the alpha heating of the plasma. The thermal excursion continues for another $8.6 \mathrm{sec}$ until $T_{i}$ reaches its steady-state operating value of $13.5 \mathrm{keV}$. After about $15 \mathrm{sec}$ into the reference burn substantial D-T burning takes place producing in excess of $1700 \mathrm{MW}$ of fusion power at a plasma beta $\left(\beta_{T}\right)$ of $16.7 \%$ which includes alphas. If one includes the additional power contribution resulting from the exothermic tritium-breeding reaction within the lithium blanket a total thermal fusion power of $\sim 2200 \mathrm{MW}$ is produced. At a thermal efficiency of $35 \%$, this prototype FCT power reactor could serve as a representative demo plant producing in excess of $750 \mathrm{MWe}$. The sequence of events leading to the steady-state operating conditions described above are briefly highlighted chronologically in Table 2 while Figs. 2 and 3 illustrate the ion and electron temperature variation during the burn and the components of the plasma power balance during startup, respectively. In looking at the steady-state temperatures in Fig. 2 it should be noted that transport due to trapped particle modes appears to be particularly beneficial in providing a means for controlling the reactivity of the plasma. Also apparent from Fig. 3 is the advantage of the low-density startup technique which can utilize even tangential injection. As the density increases to the point where beam penetration becomes marginal (at approx. $3.6 \mathrm{sec}$ ) sufficient alpha power exists to more than compensate for the decrease in beam input power (see Table 2). The above argument should be especially true if profile effects are taken into account." If the plasma is characterized by a peaked density and temperature profile, significant alpha heating power should be concentrated near the plasma center. 


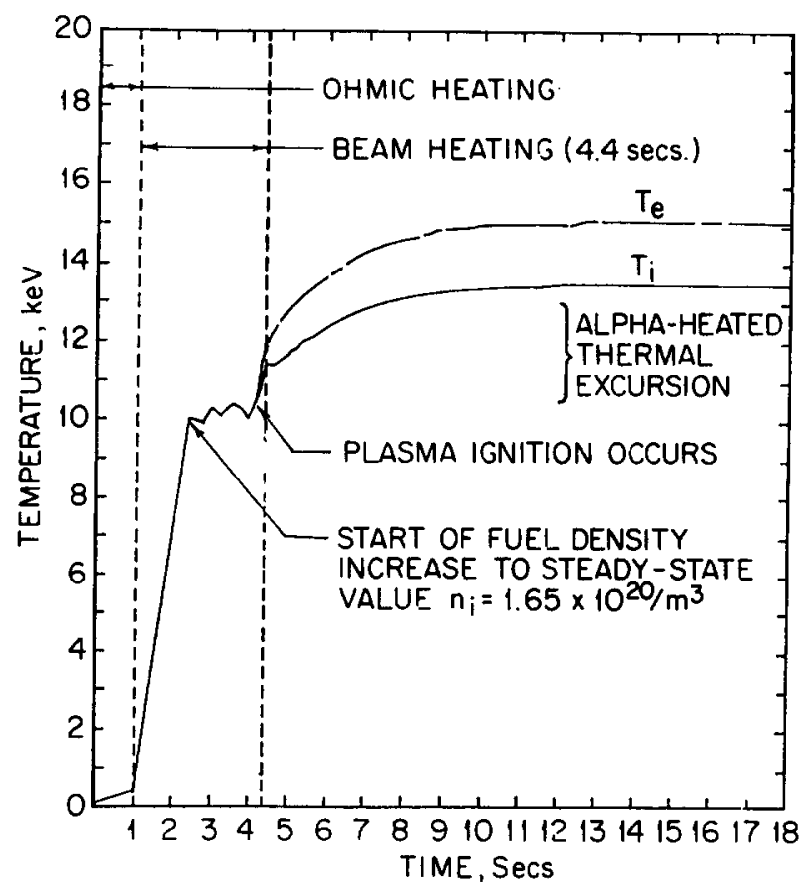

Fig. 2. Ion and electron temperature variation during burn cycle.

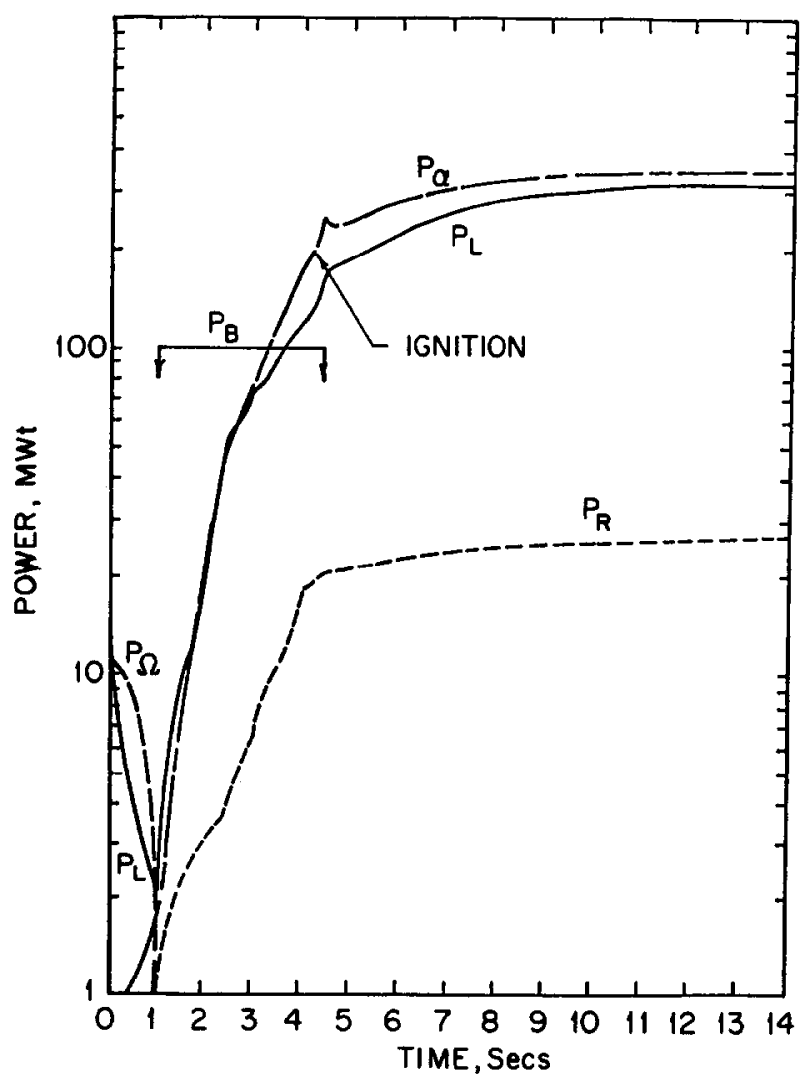

Fig. 3. Power balance components during startup. The parameters $P_{\Omega}, P_{B}, P_{a}, P_{R}$ and $P_{L}$ are the ohmic heating power, beam power, fusion alpha power and power losses due to radiation and transport, respectively. 
Table 2. Sequence of events for FCT reference burn.

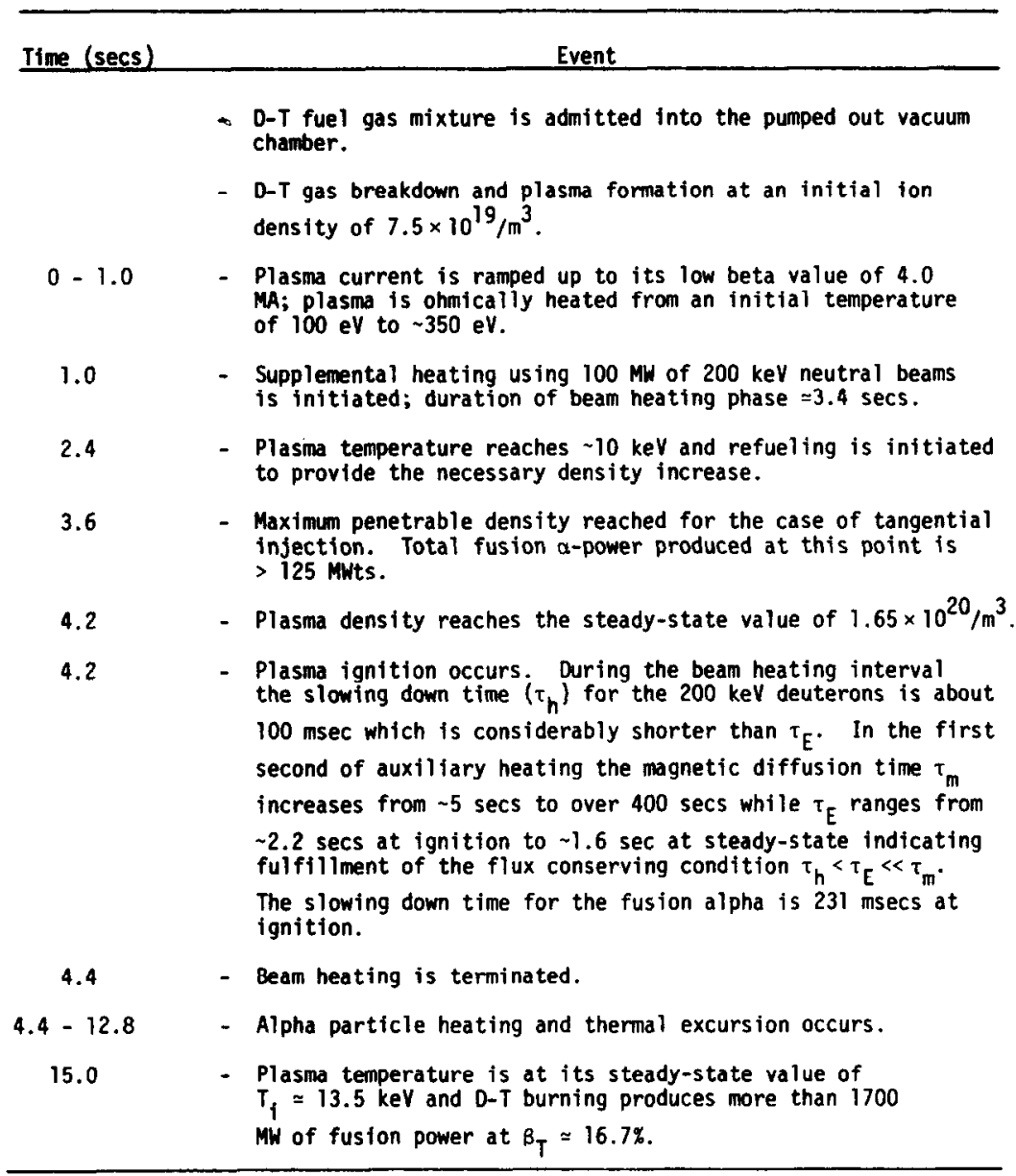

In regards to the high value for plasma $\beta_{T}$ it is necessary to comment here on the validity of the above zero dimensional (0-D) study results which are based on average temperatures and densities. Studies ${ }^{11}$ have shown that the spatial profiles for the plasma temperature and density can seriously underestimate the performance characteristic for a given size reactor when compared to 0-D results, especially if trapped particle modes are the dominant transport mechanism. It should be possible however, in the same size machines, to generate the equivalent, $0-D$ predicted fusion power by operating with sufficiently peaked density and temperature profiles. If the spatial profiles for these parameters are centrally peaked one would expect the fusion power density to be even more sharply peaked thereby producing a large percentage of the total fusion power in a fraction of the available plasma volume. The result is a lower volume-averaged beta. Recent estimates ${ }^{12}$ have been made which take this fact into account by using the peaked pressure profile characteristic of FCT operation. The results indicate that the same thermonuclear power can be produced at a $\beta_{T}$ of approximately one half of the $0-D$ value which would suggest a beta value for the FCT power reactor of around $8.5 \%$.

\section{FCT REACTOR PERFORMANCE-MAKING IT BETTER AND NOT BIGGER}

In assessing the results of the FCT systems analysis, it is highly informative to compare the performance characteristics of the FCT power system with another commercial power reactor design, UWMAK- $\mathrm{II}^{13}$ which has comparable toroidal magnetic field on axis and also assumes trapped particle mode scaling. The comparison is in some ways unfair since UWMAK-II was designed subject to the equilibrium constraints $\beta_{p} \leqslant A$. The low- $\beta$ Tokamak systems operated at low power densities and therefore required an "uncomfortably" large size to generate significant amounts of fusion power. Despite this fact the comparison does have merit because it highlights the continuing evolution of the Tokamak system. 
Table 3 summarizes the machine parameters and plasma characteristics for the two power reactors and Fig. 4 illustrates the significant reductions in the overall system which can be realized through increased power density and wall loading. The most striking feature of the FCT power reactor is the fact that its volume is about 15 times smaller than that of UWMAK-II yet it produces approximately one half of the fusion power output of UWMAK-II. The implications of FCT operation on fusion reactor design and economics should be particularly attractive to the electrical utilities.

Table 3. Comparison of UWMAK-II and FCT/prototype power reactor.

\begin{tabular}{|c|c|c|}
\hline & UWMAK-II & FCT/1.50 m Device \\
\hline Major Radius, $\mathrm{R}$ & $13.0 \mathrm{~m}$ & $6.0 \mathrm{~m}$ \\
\hline Plasma Radius, $a_{p}$ & $5.0 \mathrm{~m}$ & $1.5 \mathrm{~m}$ \\
\hline Elongation, $\sigma$ & 1.0 & 1.6 \\
\hline Aspect Ratio, A & 2.6 & 4.0 \\
\hline Plasma Volume, $v_{p}=2 \pi^{2} a^{2} \sigma R$ & $6415 \mathrm{~m}^{3}$ & $426 \mathrm{~m}^{3}$ \\
\hline Plasma Surface Area, $A_{p s}=4 \pi^{2} A a_{p}^{2} \sqrt{\frac{1+\sigma^{2}}{2}}$ & $2566 \mathrm{~m}^{2}$ & $474 m^{2}$ \\
\hline Toroidal Magnetic Field at $R, B_{T}$ & $3.57 \mathrm{~T}$ & $3.6 \mathrm{~T}$ \\
\hline $\begin{array}{l}\text { Poloidal Magnetic Field at Plasma Surface } \\
\text { (Low Beta Value), } B_{p}\end{array}$ & $0.60 \mathrm{~T}$ & $0.40 \mathrm{~T}$ \\
\hline Plasma Current (Low Beta Value), $I_{p}$ & 14.9 M.A. & 4.0 M.A. \\
\hline DT Fuel Ion Density, $n_{i}$ & $7.5 \times 10^{19} / \mathrm{m}^{3}$ & $1.65 \times 10^{20} / \mathrm{m}^{3}$ \\
\hline Impurity Level, $z_{\text {eff }}$ & 1.05 & 1.07 \\
\hline Ion Temperature, $T_{\mathbf{i}}$ & $11.9 \mathrm{keV}$ & $13.5 \mathrm{keV}$ \\
\hline Kinetic Energy Content & $3120 \mathrm{MJ}$ & $549 \mathrm{MJ}$ \\
\hline Gross Energy Confinement Time, $\tau_{E}$ & $3.9 \mathrm{sec}$ & $1.6 \mathrm{sec}$ \\
\hline Plasma Beta $(\%), \beta_{\mathrm{T}}$ & 6.4 & 16.7 \\
\hline Total Fusion Power Generated, $\mathrm{P}_{\mathrm{r}}$ & $5000 \mathrm{MW}$ & $2200 \mathrm{MW}$ \\
\hline Neutron Loading at Plasma Surface, $P_{W}$ & $1.27 \mathrm{MW} / \mathrm{m}^{2}$ & $2.92 \mathrm{MW} / \mathrm{m}^{2}$ \\
\hline Fusion Power Density, $P_{F} / V_{p}$ & $0.78 \mathrm{MW} / \mathrm{m}^{3}$ & $5.2 \mathrm{MW} / \mathrm{m}^{3}$ \\
\hline Beam Power to Plasma, $P_{B}$ & $200 \mathrm{MW}$ & $100 \mathrm{MW}$ \\
\hline Injection Energy, $U_{B}$ & $500 \mathrm{keV}$ & $200 \mathrm{keV}$ \\
\hline
\end{tabular}

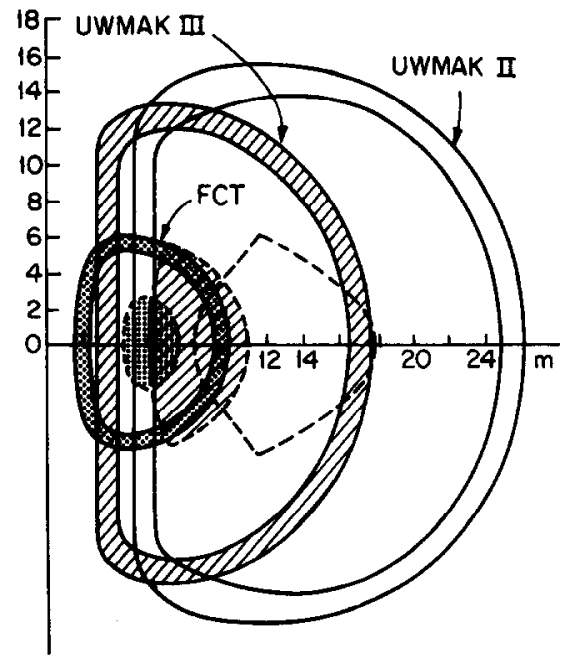

Fig. 4. Comparison of plasma and toroidal field magnet sizes in UWMAK-II, -II: and a $2200 \mathrm{MW}$ FCT power reactor. 
Perhaps more important than the comparison of two "paper" reactor studies is the fact that the FCT system may be realizable without necessitating large extrapolations both in plasma size and technology beyond that of the Tokamak Fusion Test Reactor scheduled for operation in the early 1980s. Studies on expanding radius startup ${ }^{14}$ of reactor-size plasmas indicate that deuterium neutral beams with energies of $\sim 120 \mathrm{keV}(120 \mathrm{keV}$ beams are planned for TFTR) can successfully bring an expanding radius plasma to thermonuclear burn conditions. Again, this is supported by the fact that appreciable central core heating is provided by fusion alphas.

Significant reductions in the pulsed power supply requirements may also be possible if an auxiliary plasma heating technique can eliminate the large voltage spike requirements necessary in "burning through" the low $Z$-impurity radiation barrier during startup. The proposed technique $^{15}$ of plasma initiation and preheating, via microwaves, near the upper hybrid resonance (UHR) may provide such an auxiliary technique.

\section{REFERENCES}

1. J. F. Clarke and D. J. Sigmar, Phys. Rev. Lett. 38, 70 (1977.

2. R. A. Dory and Y.-K. M. Peng, Nucl. Fusion 17. 21 (1977).

3. The EPRI Study, Impact of confinement physics on fusion reactor design and economics and the utility interface, RFP.547.

4. W. M. Stacey Jr., C. C. Baker, and M. Roberts, Proc. 6th Conf. Plasma Physics and Controlled Nuclear Research (Berchtesgaden 1976), Vol. III, p. 255. IAEA, Vienna (1977).

5. W. M. Stacey Jr. et al., Tokamak Experimental Power Reactor Studies, Argonne National Laboratory, ANL/CTR-752, June (1975).

6. S. E. Attenberger, F. B. Marcus, and D. G. McAlees, Dynamics of tokamak plasma experiments and reactors. ORNLTTM-5509, Nov. 1976.

7. J. F. Clarke, High beta flux-conserving tokamaks. ORNLTM-5429, June (1976).

8. Y.K. M. Peng and J. A. Rome, Proc. of the Plasma Heating Requirements Workshop, 5-7 Dec. 1977, CONF-771241.

9. J. W. Willis et al., Natural beam energy and power requirements for the next generation of tokamaks. ERDA $76-77$ citing $\mathrm{H}$. C. Howes, Deam Penetration and Profile Effects in Neutral Injection Heating of Tokamaks. ORMAK-TM No. 210, 15 Jan. 1976.

10. J. F. Clarke and D. G. McAlees, High beta predemonstration fusion devices (Philosophy Characteristics and R \& D Requirements), ORNL/TM-5692, Nov, 1976.

11. J. Kesner and R. W. Conn, Nucl. Fusion 16, 3(1976).

12. F. B. Marcus and S. E. Attenberger, Sensitivity study of TNS tokamak parameters. to be published p. 4, citing Y-K. M. Peng and J. A. Holmes, Energy balance estimates for TNS. internal memo, Oak Ridge National Laboratory, 17 Jan, 1977.

13. B. Badger et al., UMWAK-II, A conceptual tokamak power reactor design, Rep. No. UWFDM-112 (1975), University of Wisconsin.

14. W. A. Houlberg et al., Startup scenarios in tokamak reactors, (ORNL), ANS San Diego Meeting, 18-22 June, 1978.

15. Y-K. M. Peng and S. K. Borowski, Microwave startup of tokamak plasmas near electron cyclotron and upper hybrid resonances, ORNL/TM-6112, Dec. 1977, (to be published in Nucl. Fusion). 